Electronic Notes in Discrete Mathematics (EuroComb07), 29, pp 365-371 (2007)

\title{
Fully Optimal Bases and the Active Bijection in Graphs, Hyperplane Arrangements, and Oriented Matroids
}

\author{
Emeric GIOAN ${ }^{1}$ \\ CNRS (LIRMM, Université Montpellier 2), France \\ Michel LAS VERGNAS ${ }^{2}$ \\ CNRS (Combinatoire \& Optimisation, Université Paris 6), France
}

\begin{abstract}
In this note, we present the main results of a series of forthcoming papers, dealing with bijective generalizations of some counting formulas. New intrinsic constructions in oriented matroids on a linearly ordered set of elements establish notably structural links between counting regions and linear programming. We introduce fully optimal bases, which have a simple combinatorial characterization, and strengthen the well-known optimal bases of linear programming. Our main result is that every bounded region of an ordered hyperplane arrangement, or ordered oriented matroid, has a unique fully optimal basis, providing the active bijection between bounded regions and uniactive internal bases. The active bijection is extended to an activity preserving mapping between all reorientations and all bases of an ordered oriented matroid. It gives a bijective interpretation of the equality of two expressions for the Tutte polynomial, as well as a new expression of this polynomial in terms of beta invariants of minors. There are several refinements, such as an activity preserving bijection between regions (acyclic reorientations) and no-broken-circuit subsets, and others in terms of hyperplane arrangements, graphs, and permutations.
\end{abstract}

Keywords: oriented matroid, hyperplane arrangement, graph, Tutte polynomial, linear programming, optimal basis, bijection, reorientation, basis, region, no broken circuit.

1 Email: Emeric.Gioan@lirmm.fr

2 Email: mlv@math.jussieu.fr 


\section{Introduction}

All constructions in this note can be expressed in terms of graphs or hyperplane arrangements. However, the most general and natural language is provided by oriented matroids [1].

Let $M$ be an ordered matroid, i.e. on a linearly ordered set. By a classical theorem of W.T. Tutte for graphs, extended to matroids by H.H. Crapo, the Tutte polynomial of $M$ is $t(M ; x, y)=\sum_{i, j} b_{i, j} x^{i} y^{j}$ where $b_{i, j}$ is the number of bases of $M$ such that $i$ basis elements are smallest in their fundamental cocircuit and $j$ non-basis elements are smallest in their fundamental circuit. The parameters $i$ and $j$ for a given basis are its internal and external activities.

Let $M$ be an ordered oriented matroid on $E$. An element of $E$ is dual-active resp. active in $M$ if it is the smallest element of a positive cocircuit resp. circuit of $M$. The dual-orientation resp. orientation activity of $M$ is the number of dual-active resp. active elements of $M$. M. Las Vergnas has shown in [15] that $t(M ; x, y)=\sum_{i, j} o_{i, j} 2^{-i-j} x^{i} y^{j}$ where $o_{i, j}$ is the number of subsets $A$ of $E$ such that the oriented matroid $-{ }_{A} M$, obtained by reorientation of $M$ on $A$, has dual-orientation activity $i$ and orientation activity $j$. This second formula contains several results of the literature on counting acyclic orientations in graphs, regions in arrangements of hyperplanes and pseudohyperplanes, acyclic reorientations of oriented matroids with $t(M ; 2,0)[10][12][13][14][16][17][18]$.

Comparing these two expressions for $t(M ; x, y)$, we get the orientationbasis activity formula $o_{i, j}=2^{i+j} b_{i, j}$ for all $i, j$. The question arises of a bijective interpretation [15]. The problem is to define a natural $2^{i+j}$-to- 1 activity preserving correspondence between reorientations and bases.

The series of papers [6][7][8][9] gives a complete answer to the above question. The correspondence we define, called the active correspondence, is induced by an activity preserving mapping, called the active mapping, which sends an ordered oriented matroid to one of its bases. It can be refined into an active bijection between reorientations and subsets, and, in particular, between acyclic reorientations (regions) and subsets with no broken circuit.

There are several ways to construct the active correspondence. A first construction, probably the most interesting one, presented in this note, reduces the problem to the uniactive internal case [6][8] - the bounded case, from a geometrical point of view - by using duality, and decomposing activities [7]. A second construction is by means of inductive deletion/contraction relations [9]. Several particular cases with specific properties have already been published: uniform and rank-3 oriented matroids [3], graphs [4], and supersolvable hyperplane arrangements [5]. 


\section{The bounded case}

We first consider the case $o_{1,0}=2 b_{1,0}(=2 \beta)$ of the orientation-basis activity formula. In a real hyperplane arrangement this formula is a result of $\mathrm{T}$. Zaslavsky [18], generalized to oriented matroids by M. Las Vergnas [13].

Let $M$ be an ordered oriented matroid on $E=\left\{e_{1}, e_{2}, \ldots, e_{n}\right\}<$ with rank $r$. The smallest nonloop element $p$ of $M$ is called the (pseudohyper)plane at infin$i t y$. In this section, we assume that $M$ is loopless and $p=e_{1}$. If $M$ is acyclic, then it is bounded if every positive cocircuit contains $p$, i.e. it has orientation activity 0 and dual-orientation activity 1 . Bounded acyclic reorientations are bounded regions in the topological representation of an oriented matroid. An acyclic digraph is bounded if it is bipolar at the edge $p$, i.e. it has a unique source and a unique sink, vertices of $p$.

Let $B$ be a basis of $M$. For $e \notin B$, the fundamental circuit of $e$ with respect to $B$, denoted by $C(B ; e)$, is the unique circuit contained in $B \cup\{e\}$, with $e$ signed + . For $b \in B$, the fundamental cocircuit of $b$ with respect to $B$, denoted by $C^{*}(B ; b)$, is the unique cocircuit contained in $(E \backslash B) \cup\{b\}$, with $b$ signed + . Note that if $b \in C(B ; e)$ then $\{b, e\}=C(B ; e) \cap C^{*}(B ; b)$. A basis $B$ of $M$ is internal if no element $e \in E \backslash B$ is minimal in $C(B ; e)$. An internal basis $B$ of $M$ is uniactive if the minimal element of any fundamental cocircuit of $b \in B$ with respect to $B$ is $p$. Hence a uniactive internal basis has external activity 0 and internal activity 1 . The problem is thus to construct a natural bijection between bounded regions (on positive side of $p$ ) and uniactive internal bases.

The (fundamental) tableau of a basis $B$ is an $n \times n$ matrix with coefficients in $\{+,-, 0\}$. The $i$-th column is $C^{*}\left(B ; e_{i}\right)$ if $e_{i} \in B$, the $i$-th row is $-C\left(B ; e_{i}\right)$ if $e_{i} \notin B$, and coefficients are 0 everywhere else.

Definition. We say that a basis $B$ of $M$ is fully optimal if the first nonzero sign of each row of its tableau is + and the first nonzero sign of each column, except the first one, is -.

Equivalently, with $B=\left\{b_{1}, b_{2}, \ldots, b_{r}\right\}_{<}$and $E \backslash B=\left\{c_{1}, c_{2}, \ldots, c_{n-r}\right\}_{<}$, the basis $B$ of $M$ is fully optimal if and only if $B$ is uniactive internal, the covector $C^{*}\left(B ; b_{1}\right) \circ C^{*}\left(B ; b_{2}\right) \circ \cdots \circ C^{*}\left(B ; b_{r}\right)$ is positive, and the vector $C\left(B ; c_{1}\right) \circ C\left(B ; c_{2}\right) \circ \cdots \circ C\left(B ; c_{n-r}\right)$ has $p=e_{1}=b_{1}$ as its unique negative element. Note that if $M$ has a fully optimal basis then it is bounded acyclic.

Theorem 1. [6] A bounded acyclic ordered oriented matroid $M$ has exactly one fully optimal basis, denoted by $\alpha(M)$. The active mapping $\alpha$ induces a bijection between bounded acyclic reorientations of $M$, on the positive side of $p$, and uniactive internal bases of $M$. 
From uniactive internal bases to bounded acyclic reorientations, the bijection is easy to build [6] by choosing successively, with respect to the linear ordering, the reorientation of the elements, i.e. the signs in the (unsigned) tableau of the basis, so that the above definition is satisfied. The converse construction can be done by means of refinements of linear programming [8].

Fully optimal bases are a strengthening of the optimal bases considered in linear programming, and, more generally, in oriented matroid pseudolinear programming [1]. By the Simplex Criterion, a basis is optimal in the program $(M ; p, f)$, with plane at infinity $p$ and objective function $f$, if and only if its tableau is such that the column $p$ has only + signs and the row of $f$ has only - signs, except maybe on $p$. Hence, $M$ being bounded acyclic and ordered, and $\left\{p, f_{1}, f_{2}, \ldots, f_{r-1}\right\}<$ being the minimal basis in the induced lexicographic ordering, the fully optimal basis of $M$ is an optimal basis of the pseudolinear program $\left(M ; p, f_{1}\right)$. Note that $\alpha(M)$ contains $p$ but not $f_{1}$ since it is uniactive internal. An optimal vertex for the program is the fundamental cocircuit of $p$ with respect to an optimal basis. Several optimal vertices may exist, and several optimal bases may produce the same optimal vertex. Hence, optimal bases of bounded regions are generally not unique.

The fully optimal basis of a given bounded region is, on the contrary, always unique. It can be computed by an algorithm consisting of a sequence of (at most) $r-1$ linear programs of a generalized type. The precise construction from [8] cannot be detailed in this note. Briefly, the fully optimal basis is equivalent to an optimal flag of $r$ flats, corresponding to successive compositions of its fundamental cocircuits (instead of one flat in usual linear programming), with maximality properties with respect to the $r-1$ independent objective functions $\left\{f_{1}, f_{2}, \ldots, f_{r-1}\right\}_{<}$(instead of one objective function).

Fully optimal bases also have a duality property extending the duality of linear programming [8], and a deletion/contraction construction extending the usual LP construction by variable/constraint deletion [9].

An example of the active bijection is shown in the figure. Consider only bounded regions. In each one is written its fully optimal uniactive internal basis $B=\{p, e, f\}_{<}$. The two covectors $C^{*}(B ; p)$ and $C^{*}(B ; p) \circ C^{*}(B ; e)$ are indicated as a bold vertex and an incident segment. The bold vertex is the optimal vertex of a multiprogram $\left(M ; p, f_{1}, f_{2}\right)$ : it is the farthest from $f_{1}$ in the region, and the farthest from $f_{2}$ in faces parallel to $f_{1}$. The pseudoline $e$ is the smallest containing this vertex. And the segment, supported by $f$, is obtained by a flag refinement of LP, not precisely described here, consisting, roughly, in optimizing mobile segments around the fixed optimal vertex. 


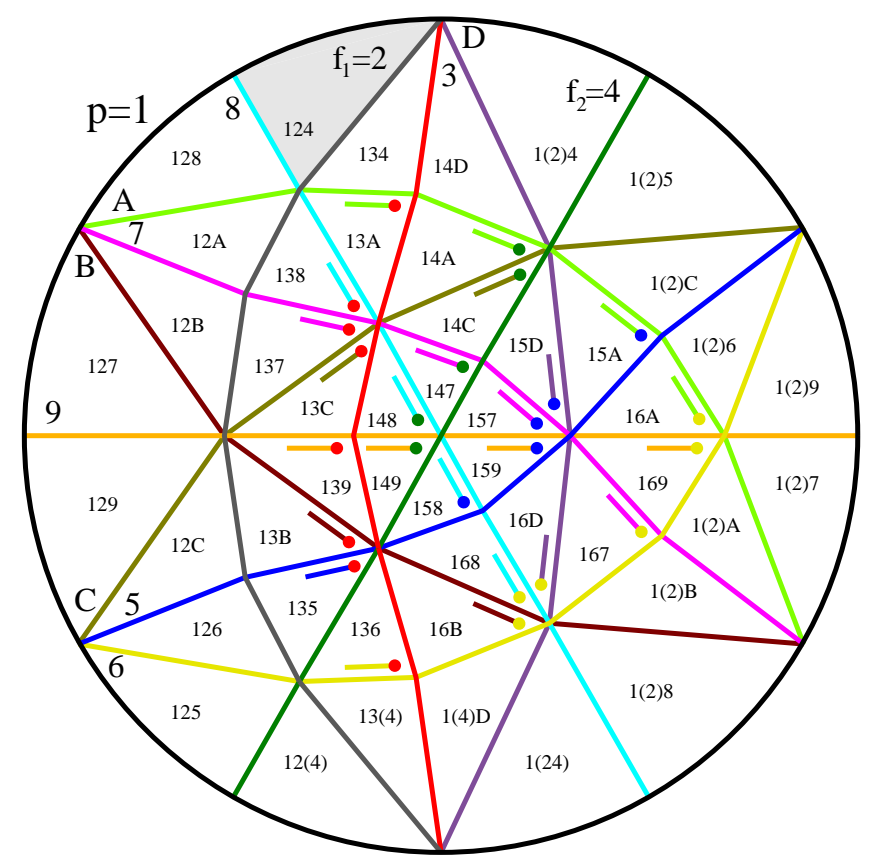

\section{The general case}

Definition. The active mapping $\alpha$ is extended to general ordered oriented matroids by the two following characteristic properties: 1) $\alpha\left(M^{*}\right)=E \backslash \alpha(M)$, and 2) $\alpha(M)=\alpha(M / A) \cup \alpha(M(A))$ where $A$ is the union of all positive circuits of $M$ whose smallest element is the greatest active element of $M$.

In property 2), if $A \neq \emptyset$, then $M(A)$ has a bounded acyclic dual, and $M / A$ has one active element less than $M$. Applying recursively the two properties, in any way, to $M$ with $i$ dual-active elements and $j$ active elements, we build a well defined sequence of subsets $\emptyset=F_{j}^{\prime} \subset \ldots \subset F_{0}^{\prime}=F_{c}=F_{0}^{\prime \prime} \subset \ldots \subset F_{i}^{\prime \prime}=E$, where $F_{c}$ is a cyclic flat of $M$, and the $i+j$ minors $M_{k}^{\prime \prime}=M\left(F_{k}^{\prime \prime}\right) / F_{k-1}^{\prime \prime}$, $1 \leq k \leq i$, and $M_{k}^{\prime}=M\left(F_{k-1}^{\prime}\right) / F_{k}^{\prime}, 1 \leq k \leq j$, are respectively bounded acyclic or dually bounded acyclic. Thus, $\alpha(M)$ is well defined as the union of the images of these minors under $\alpha$, and $\alpha(M)$ is a basis of $M$. This increasing sequence of subsets is called the active decomposing sequence of $M$. The successive differences (i.e. the subsets $A$ ) form the active partition of $M$. The set of $2^{i+j}$ reorientations of $M$ obtained by reorienting arbitrary parts in the active partition is called the activity class of $M$. Activity classes form a partition of the set of reorientations of $M$. All reorientations in an activity class have the same image under $\alpha$, and the same active decomposing sequence.

Theorem 2. [7] Let $M$ be an ordered oriented matroid, $\alpha$ induces an activity preserving bijection between activity classes of reorientations and bases of $M$. 
Some active decomposing sequences, preserved by $\alpha$, can also be described for bases [7]. Numerically, this decomposition extends the known formula $t(M ; x, y)=\sum t(M / F ; x, 0) t(M(F) ; 0, y)$, summed over cyclic flats $F$ of $M$, implicit in [2] by an explicit bijection, and called the convolution formula in [11]. We get the corollary below, where, by convention, $\beta(M)=1$ if $|E|=1$.

Corollary 3. [7] Let $M$ be an ordered matroid on E.

$t(M ; x, y)=\sum\left(\prod_{1 \leq k \leq i} \beta\left(M\left(F_{k}^{\prime}\right) / F_{k-1}^{\prime}\right)\right)\left(\prod_{1 \leq k \leq j} \beta\left(M\left(F_{k-1}^{\prime \prime}\right) / F_{k}^{\prime \prime}\right)\right) x^{i} y^{j}$ where the sum is over all active decomposing sequences of bases of $M$.

The correspondence induced by $\alpha$ in Theorem 2 depends only on the reorientation class of $M$. The figure illustrates the correspondence between regions and internal bases. The basis 134 (resp. 14D) is the image of two regions (on represented side of 1$)$, having active partition $123 D+(E \backslash 123 D)$, it is the union of the unicative internal bases 13 (resp. $1 D$ ) of $M(123 D$ ), and 4 of $M / 123 D$.

A refinement, depending this time on $M$, uses the classical partition of $2^{E}$ into intervals $[B \backslash \operatorname{Int}(B), B \cup \operatorname{Ext}(B)]$ where $B$ is a basis with internally active elements $\operatorname{Int}(B)$ and externally active elements $\operatorname{Ext}(B)$. An interval has cardinality $2^{i+j}$ and corresponds to $2^{i+j}$ reorientations. Hence, by reorienting elements of $\operatorname{Int}(B) \cup \operatorname{Ext}(B)$, we get the active bijection between reorientations and subsets. Precisely, for $A \subseteq E$ and $B=\alpha\left(-{ }_{A} M\right)$, it is defined by $\bar{\alpha}_{M}(A)=$ $B \backslash(A \cap \operatorname{Int}(B)) \cup(A \cap \operatorname{Ext}(B))$. In particular, considering only internal bases for which $\operatorname{Ext}(B)=\emptyset$, the elements of intervals $[B \backslash \operatorname{Int}(B), B]$ are exactly the no-broken-circuit subsets, and are in active bijection with acyclic reorientations, i.e. with regions. The figure shows an example of this active bijection, by considering fundamental grey region, and deleting elements in brackets. Several active bijections derived from these general constructions are listed in the following table.

\begin{tabular}{|c|c|c|c|}
\hline structure & \multicolumn{2}{|c|}{ active bijection } & ref. \\
\hline oriented matroids & $\begin{array}{l}\text { activity classes of reorientations } \\
\text { act. cl. of acyclic reorientations } \\
\text { act. cl. of totally cyclic reor. } \\
\text { bounded acyclic reorientations } \\
\text { reorientations } \\
\text { acyclic reorientations }\end{array}$ & $\begin{array}{c}\text { bases } \\
\text { internal bases } \\
\text { external bases } \\
\text { uniactive internal bases } \\
\text { subsets } \\
\text { no-broken-circuit subsets }\end{array}$ & $\begin{array}{l}{[6]-[9]} \\
{[6]-[9]} \\
{[6]-[9]} \\
{[6]-[9]} \\
{[6]-[9]} \\
{[6]-[9]}\end{array}$ \\
\hline $\begin{array}{c}\text { hyperplane } \\
\text { arrangements }\end{array}$ & $\begin{array}{c}\text { reorientations }=\text { signatures } \\
\text { acyclic reorientations }=\text { regions }\end{array}$ & bases $=$ simplices & $\begin{array}{l}6]-[9] \\
{[5]-[9]}\end{array}$ \\
\hline graphs & $\begin{array}{c}\text { reorientations }=\text { orientations } \\
\text { unique sink acyclic orientations } \\
\text { bipolar orientations }\end{array}$ & $\begin{array}{c}\text { bases }=\text { spanning trees } \\
\text { internal spanning trees } \\
\text { uniactive internal spanning trees }\end{array}$ & $\begin{array}{c}4]-[9] \\
{[4]} \\
{[4]}\end{array}$ \\
\hline uniform o.m. & bounded regions & LP optimal vertices & {$[3]$} \\
\hline $\begin{array}{l}\text { supersolvable } A_{n} \\
\text { supersolvable } B_{n}\end{array}$ & $\begin{array}{c}\text { permutations } \\
\text { signed permutations }\end{array}$ & $\begin{array}{c}\text { increasing trees } \\
\text { signed increasing trees }\end{array}$ & $\begin{array}{c}{[4][5]} \\
{[5]}\end{array}$ \\
\hline
\end{tabular}




\section{References}

[1] Björner, A., M. Las Vergnas, B. Sturmfels, N. White, and G. Ziegler, "Oriented Matroids", 2nd ed., Cambridge University Press, Cambridge, 1999.

[2] Etienne, G., and M. Las Vergnas, External and internal elements of a matroid basis, Discrete Math. 179 (1999), 111-119.

[3] Gioan, E., and M. Las Vergnas, Bases, reorientations and linear programming in uniform and rank-3 oriented matroids, (Special issue Workshop on Tutte polynomials, Barcelona 2001) Adv. in Appl. Math. 32 (2004), 212-238.

[4] Gioan, E., and M. Las Vergnas, Activity preserving bijections between spanning trees and orientations in graphs, (Special issue FPSAC 2002) Discrete Math. 298 (2005), 169-188.

[5] Gioan, E., and M. Las Vergnas, The active bijection between regions and simplices in supersolvable arrangements of hyperplanes, (Stanley Festschrift) Electronic Journal of Combinatorics 11(2) (2006) \#R30.

[6] Gioan, E., and M. Las Vergnas, The active bijection in graphs, hyperplane arrangements, and oriented matroids 1. The fully optimal basis of a bounded region, to appear

[7] — 2. Decomposition of activities, to appear.

[8] — 3. Linear programming construction of fully optimal bases, in preparation.

[9] — 4. Deletion/contraction constructions and universality, in preparation.

See www.lirmm.fr/ gioan, www.ecp6.jussieu.fr/pageperso/las_vergnas

[10] Greene, C., and T. Zaslavsky, On the interpretation of Whitney numbers through arrangements of hyperplanes, zonotopes, non-Radon partitions and orientations of graphs, Trans. Amer. Math. Soc. 280 (1983), 97-126.

[11] Kook, W., V. Reiner, and D. Stanton, A convolution formula for the Tutte polynomial, J. Comb. Theory Ser. B 76(2) (1999), 297-300.

[12] Las Vergnas, M., Matrö̈des orientables, C. R. Acad. Sci. Paris Sér. A 280 (1975), 61-64

[13] Las Vergnas, M., Acyclic and totally cyclic orientations of combinatorial geometries, Discrete Math. 20 (1977/78), 51-61.

[14] Las Vergnas, M., Convexity in oriented matroids, J. Combin. Theory Ser. B 29 (1980), 231-243.

[15] Las Vergnas, M., The Tutte polynomial of a morphism of matroids II. Activities of orientations, "Progress in Graph Theory" (Proc. Waterloo Silver Jubilee Conf. 1982), Academic Press, Toronto, 1984, 367-380.

[16] Stanley, R.P., Acyclic orientations of graphs, Discrete Math. 5 (1973), 171-178.

[17] Winder, R.O., Partitions of N-space by hyperplanes, SIAM J. Applied Math. 14 (1966), 811-818.

[18] Zaslavsky, T., "Facing up to arrangements: Face count formulas for partitions of space by hyperplanes", Mem. Amer. Math. Soc., No. 154, Amer. Math. Soc., Providence, 1975. 